Pol. J. Food Nutr. Sci., 2022, Vol. 72, No. 1, pp. 101-108

On-line ISSN: 2083-6007

Print ISSN: $1230-0322$

DOI: $10.31883 /$ pjfns/146562 http://journal.pan.olsztyn.pl

Original article

Section: Food Quality and Functionality

\title{
Antioxidative Capacity of Soyfoods and Soy Active Compounds
}

\author{
Wanida T. Chitisankul ${ }^{*}$ (1), Kazuko Shimada², Chigen Tsukamoto ${ }^{3}$ (D)
}

\author{
${ }^{I}$ Nutrition and Health, Institute of Food Research and Product Development, Kasetsart University, 50 Ngamwongwan Rd., \\ Chatuchak, Bangkok, 10900, Thailand \\ ${ }^{2}$ Faculty of Health Science, Hiroshima Shudo University, 1-1-1 Ozukahigashi, Asaminami-ku, Hiroshima, 731-3195, Japan \\ ${ }^{3}$ Faculty Agriculture, Iwate University, 3-18-8 Ueda, Morioka, Iwate, 020-8550, Japan
}

Key words: soybean, ORAC, isoflavone, peroxyl radical scavenging, soy saponin

Soyfood isoflavones and soyasaponins are effective compounds in terms of their health-promoting properties. Their chemical structure plays an important role in their antioxidative activity. Thus, six isoflavones and four soyasaponins that are targeted in soyfood were evaluated for their peroxyl radical scavenging capacities by the hydrophilic-oxygen radical absorbance capacity (H-ORAC) method. The antioxidant capacity of non-fermented and fermented soyfoods was also determined by the same method. The results revealed that isoflavones showed higher peroxyl radical scavenging capacities than soyasaponin, with their activities found to depend on their chemical structure. The aglycone isoflavones promoted higher H-ORAC values than glycoside and malonyl glycoside isoflavones, respectively. On the other hand, DDMP saponin promoted a higher H-ORAC value than its derived compound, group B saponin, and the aglycone soyasaponin. In the case of soyfoods, fermented soyfoods had higher antioxidative capacity that the non-fermented ones, especially the long-term fermented products. Soybean-koji miso presented the highest H-ORAC value, followed by natto, soy sauce, and tempeh. Moreover, lightness $\left(L^{*}\right)$ of miso and soy sauce showed a negative correlation with H-ORAC value probably due to browning substances which might derive from the amino-carbonyl reaction. Considering the high antioxidant capacity of fermented soyfoods, it might relate to aglycone isoflavones which promote strong radical scavenging capacity. Thus, fermented soyfoods, especially miso and natto, could be considered as health-promoting foods.

\section{INTRODUCTION}

A peroxyl radical was reported as a cause of non-communicable diseases, such as cardiovascular disease, cancer, and neurological disease [Pizzino et al., 2017]. As its excess in vivo and lifestyle diseases, such as arteriosclerosis, are related, food that includes antioxidative components is strongly recommended to be consumed for these diseases prevention. Soybean is a familiar plant-based protein food component to people in Japan and Southeast Asia which additionally promotes the antioxidative effect due to its functional ingredients, such as isoflavones [Han et al., 2009; Kim et al., 2022], saponins [Yoshiki et al., 2001; Yoshiki \& Okubo, 1995], tocopherols [Carrera \& Seguin, 2016], anthocyanins [Kähkönen \& Heinonen, 2003; Zilic et al., 2019], and proanthocyanins [Xu et al., 2017]. However, rather than consuming soybeans as fresh, almost all of them are consumed as processed products. These soyfoods, e.g. miso [Matsuo \& Hitomi, 2007], soy sauce [Long et al., 2000], natto [Ping et al., 2012], and tempeh [Chang et al., 2009; Kameda et al., 2018], have also been reported as a source of natural antioxidants. The thermal processes, as well as pressurization and fermentation are used to obtain soyfoods. They could induce degradation and/or conversion of soybean native active ingredients and, in consequence, affect their functionalities [Chitisankul et al., 2015; 2021; Khosravi \& Razavi, 2021; Ping et al., 2012].

The antioxidant properties of foods and food ingredients have been measured by various methods, including the hydrophilic-oxygen radical absorbance capacity (H-ORAC) assay [Ou et al., 2001], which is an in vitro method for determining the antioxidative capacity by measuring peroxyl radical-scavenging activity of water-soluble substances [Zhong \& Shahidi, 2015]. Isoflavones [Han et al., 2009; Kim et al., 2022], 2,3-dihydro-2,5-dihydroxy-6-methyl-4H-pyran-4-one (DDMP) saponins [Yoshiki et al., 2001; Yoshiki \& Okubo, 1995], peptides [Sanjukta \& Rai, 2016; Tonolo et al., 2020], and browning substances [Ando et al., 2003] are the main water-soluble antioxidants which could be extracted by the aqueous ethanol solution from soybeans and processed soybean foods. The isoflavones contained in soybeans undergo demalonylation and further aglycone conversion by cooking and processing [Toda et al., 2000].

\footnotetext{
* Corresponding Author:

E-mail: wanida.te@ku.ac.th (W.T. Chitisankul)
} 
Their antioxidative activity was evaluated by the low-density lipoprotein (LDL) oxidation method and it had been shown that aglycone isoflavones promote higher antioxidative activity than glycosides [Lee et al., 2005]. On the other hand, the antioxidative activity of isoflavones was evaluated by the ABTS method [Ruiz-Larrea et al., 1997] and the liposome oxidation method [Arora et al., 1998], which showed no significant differences between the properties of glycoside isoflavones and their aglycones. In addition, DDMP saponins were reported to promote antioxidative capacity [Yoshiki et al., 2001; Yoshiki \& Okubo, 1995]. But it also was reported that DDMP saponin can be degraded by heating, fermenting, and aging [Chitisankul et al., 2015; Omizu et al., 2011]. However, there is no report about an evaluation of the antioxidant capacity of individual isoflavones, soyasaponins, and their derived compounds by the same methodology including several processed soybeans. Therefore, this research aimed to evaluate the peroxyl radical scavenging capacities of isoflavones, soyasaponin, and several soyfood samples by the H-ORAC assay.

\section{MATERIAL AND METHODS}

\section{Samples and reagents}

Soyfoods were purchased from food supermarkets in Morioka (Iwate, Japan). The samples included non-fermented soyfoods (NFS) and fermented soyfoods (FS) as shown in Table 1. The 11 samples of six kinds of NFS included steamed soybean, young soybean, soymilk, soy beverage, tofu, and fried tofu. The nine samples of four kinds of FS included natto, tempeh, miso, and soy sauce.

All standard reagents were of HPLC grade. They included six isoflavone standard reagents: malonyldaidzin, malonylgenistin, daidzin, genistin, daidzein, and genistein (Wako Pure Chemical Industries, Ltd., Osaka, Japan); and soyasaponin standard reagents: soyasapogenol A and soyasapogenol B (Koshiro Company Ltd., Osaka, Japan). The 2,2-azobis (2-amidinopropane) dihydrochloride (AAPH) (Wako Pure Chemical Industries, Ltd., Osaka, Japan), fluorescein sodium salt (Sigma Aldrich, Tokyo, Japan), and 6-hydroxy-2,5,7,8-tetramethylchroman-2-carboxylic acid (Trolox) (Tokyo Chemical Industry Co., Ltd., Tokyo, Japan) were analytical grade reagents.

\section{Isoflavone and saponin preparation}

Six isoflavones and soyasaponins, soyasapogenol A and soyasapogenol B, were dissolved and mixed well in a 70\% $(v / v)$ aqueous ethanol solution by vortexing or ultrasonication with the final concentration of $500 \mu \mathrm{M}$ or $1 \mathrm{mM}$ solution, respectively. DDMP saponin $\beta \mathrm{g}$ was extracted and purified from soybean hypocotyl [Chitisankul et al., 2015]. Purified DDMP saponin $\beta \mathrm{g}$ and group $\mathrm{B}$ saponin $\mathrm{Bb}$ were analyzed using HPLC [Chitisankul et al., 2019], and the purity of the purified saponin was $95 \%$ or higher. The purified DDMP saponin solution was composed of $852.1 \mu \mathrm{M}$ saponin $\beta \mathrm{g}$ and the group B saponin solution was composed of $1118 \mu \mathrm{M}$ saponin $\mathrm{Bb}$.

\section{Sample preparation}

Liquid soyfoods were directly extracted by 10 -fold volume $(w / v)$ of a $70 \%(v / v)$ aqueous ethanol solution.
TABLE 1. Code, name, and description of soyfoods including non-fermented (NF) and fermented (F) products.

\begin{tabular}{|c|c|c|}
\hline Code & Sample name & Sample description \\
\hline NF1 & Steamed soybean & Steamed whole seeds \\
\hline NF2 & Edamame & $\begin{array}{l}\text { Heated and frozen green } \\
\text { (young) soybean }\end{array}$ \\
\hline NF3 & Soymilk & Regular type soymilk \\
\hline NF4 & Soymilk w/o LOX & $\begin{array}{l}\text { Soymilk from lipoxygenase } \\
\text { enzyme deficient soybean }\end{array}$ \\
\hline NF5 & Modified soymilk & Blended soymilk \\
\hline NF6 & Soybean beverage & $\begin{array}{l}\text { Beverage from whole soybean } \\
\text { (including okara) }\end{array}$ \\
\hline NF7 & Black soybean beverage & $\begin{array}{l}\text { Beverage from whole black } \\
\text { soybean (including okara) }\end{array}$ \\
\hline NF8 & Momen tofu & Regular type tofu \\
\hline NF9 & Silken tofu & Very soft tofu \\
\hline NF10 & Abura-age & $\begin{array}{l}\text { Twice-fried tofu, deep } \\
\text { fried thin sliced tofu }\end{array}$ \\
\hline NF11 & Nama-age & Deep fried tofu \\
\hline $\mathrm{F} 1$ & Natto & Regular type natto \\
\hline $\mathrm{F} 2$ & Black soybean natto & Black soybean natto \\
\hline F3 & Tempe & $\begin{array}{l}\text { Fermented soybean } \\
\text { with Rhizopus spp. }\end{array}$ \\
\hline $\mathrm{F} 4$ & Rice miso, sweet - white & Rice-koji miso: sweet-white type \\
\hline F5 & Rice miso, light yellow & Rice-koji miso: light yellow type \\
\hline F6 & Barley miso & Barley-koji miso \\
\hline F7 & Soy miso & Soybean-koji miso \\
\hline F8 & Dark soy sauce & Dark soy sauce \\
\hline F9 & Light soy sauce & Light soy sauce \\
\hline
\end{tabular}

The sample solution was homogenized (15,500 rpm, $1 \mathrm{~min}$, Polytron homogenizer, Jakarta, Indonesia) and left to stand for $1 \mathrm{~h}$ with every $10 \mathrm{~min}$ vortex mixing. The supernatant was obtained by centrifugation at $27,000 \times g$ and $15^{\circ} \mathrm{C}$ for 15 min (Kubota 7780 centrifuge, Tokyo, Japan) as a crude extract, and then filtered with a $0.45 \mu \mathrm{m}$ membrane filter. For all tofu samples, the outer part was cut off with a ceramic knife, then the samples were fined in a mortar. Steamed soybean, edamame, natto, tempeh, and miso were crushed with a mallet and then fined in a mortar. Each solid soyfood sample was extracted in the same manner as liquid samples. The crude extracts were kept in the opaque bottles at $-20^{\circ} \mathrm{C}$ until analysis.

\section{Color measurement}

For miso and soy sauce, CIE- $L^{*}$ (lightness), $a^{*}$ (green to red color), $b^{*}$ (blue to yellow color) were measured using a colorimeter (color meter Z-300A, Nippon Denshoku Industries Co., Ltd., Tokyo, Japan). Miso was packed in a Petri dish, the lid was put on, and the reflectance was measured. In addition, soy sauce was placed in a glass cell and permeation was measured. Miso was triplicate measured per sample, and soy sauce was measured once per sample. 


\section{Peroxyl radical scavenging capability evaluation}

Hydrophilic-oxygen radical absorbance capacity (H-ORAC) assay was used to measure peroxyl radical scavenging capability [Ou et al., 2001]. The standard and sample solution were diluted with phosphate buffer (75 mM, pH 7.0), then the Trolox standard solution of $6.25 \mu \mathrm{M}$ to $100 \mu \mathrm{M}$ was used as a standard curve. The H-ORAC values of isoflavone and soyasaponin were reported as mol Trolox equivalent (TE)/mol and those of the processed soybean food as mmol Trolox equivalent (TE)/100 g sample. A fluorescence plate reader (Bio-Tek FL600, Bio-Tek Instruments, Inc., Winooski, VT, USA) was used for measurements.

\section{Statistical analysis}

Analysis of variance (ANOVA) of the experimental data was performed and the least significant difference was evaluated by Tukey's test at a 95\% confidence interval. The correlation coefficient (r) of the experimental data was analyzed. All analyses were repeated in triplicate.

\section{RESULTS AND DISCUSSION}

\section{Peroxyl radical scavenging capacity of isoflavones and soyasaponins}

The peroxyl radical scavenging capacity of isoflavones and soyasaponins had been shown as H-ORAC values (mol TE/mol) in Figure 1. Isoflavones presented higher antioxidative capacity than soyasaponins. The chemical structure of active ingredients promoted different activities. For isoflavones, high H-ORAC values presented in the order of aglycone form then glycoside form, and malonyl glycoside form. Daidzein had the highest antioxidant ability, with the $\mathrm{H}$ ORAC value reaching $9.94 \pm 0.45 \mathrm{~mol} \mathrm{TE} / \mathrm{mol}$, followed by genistein, genistin, daidzin, and malonyl glycoside isoflavone, respectively. The result was supported by a previous report which revealed that the antioxidative activity of aglycones (daidzein, genistein) was higher than that of glycosides (daidzin, genistin) depending on the method of measuring the lag time of LDL oxidation (lag time assay) [Lee et al., 2005]. Recently, Kim et al. [2022] also found that the ABTS ${ }^{*+}$ scavenging activity and ferric-reducing antioxidant power (FRAP) of soy isoflavones as aglycone were higher than those of their glycosides, and malonyl glycoside derivatives had the lowest

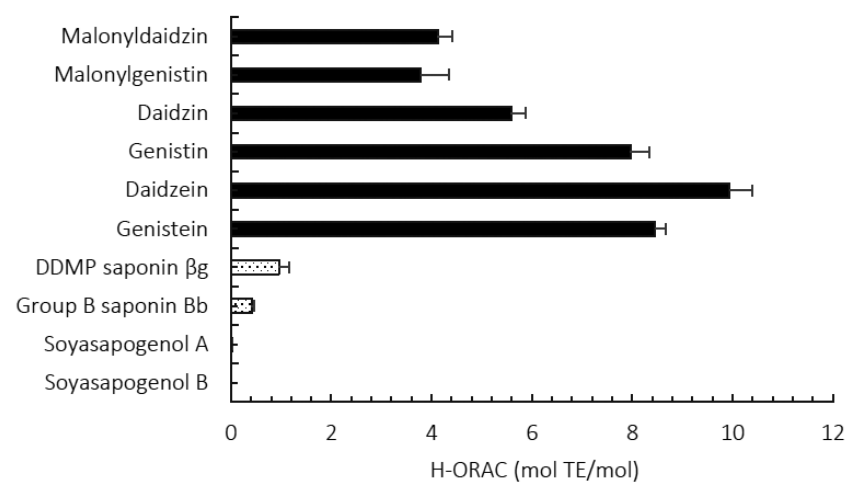

FIGURE 1. Hydrophilic-oxygen radical absorbance capacity (H-ORAC) of isoflavones and saponins. activity. Isoflavones are generally found as glycoside forms in natural sources, but their bioavailability is lower compared to aglycones [Kim et al., 2022; Setchell et al., 2002]. However, the glycoside isoflavone would be converted to aglycone by gut microflora [Bultosa, 2016].

On the other hand, high H-ORAC values of soyasaponins are presented in the order of DDMP glycoside (DDMP saponin $\beta \mathrm{g}$ ) then glycoside (group B saponin $\mathrm{Bb}$ ), and aglycone (soyasapogenol A, soyasapogenol B) (Figure 1). According to this statement, it clearly shows that the changing chemical structure affects the antioxidant activity of the compound. The degradation of DDMP saponin resulted in antioxidant activity reduction, which was agreeable with other reports [Yoshiki et al., 2001; Yoshiki \& Okubo, 1995]. The reports also revealed that the DDMP site is involved in superoxide scavenging ability [Yoshiki et al., 2001; Yoshiki \& Okubo, 1995], thus group B saponin Bb which lacks the DDMP site will not present scavenging activity. Following the evaluation of soyasaponin peroxyl radical scavenging activity in this research, DDMP saponin $\beta$ g presented strong peroxyl radical scavenging activity while group B saponin Bb presented low peroxyl radical scavenging activity. These results suggest that DDMP saponin $\beta \mathrm{g}$ can scavenge both peroxyl radicals and superoxide. Although, group B saponin Bb cannot scavenge superoxide [Yoshiki et al., 2001; Yoshiki \& Okubo, 1995], it can eliminate peroxyl radical. On the other hand, the aglycone saponins: soyasapogenol A and soyasapogenol $\mathrm{B}$, showed no scavenging activity against peroxyl radicals (Figure 1). From the above findings, it was speculated that the peroxyl radical scavenging ability was related to the DDMP site and sugar chain portion of soyasaponin. Nevertheless, DDMP saponin could be naturally found in fresh soybean or low processed soybean products which might vary depending on soybean variety and processing treatment [Chitisankul et al., 2019, 2021].

\section{Peroxyl radical scavenging capacity of non-fermented soyfoods}

The nutraceutical property due to the antioxidative capacity of 11 non-fermented soyfoods (NFS) differed and depended on the type of products (Figure 2), namely to their processing treatments. The NFS products could be categorized into three major groups: (1) steamed soybean (NF1) and frozen boiled edamame (NF2) as low-processed products, (2) soymilk or soy beverages (NF3-NF7), and (3) tofu (NF8-NF11). Only heat processes were applied to obtain the products of the first group whilst several treatments such soaking, extraction, and heating were required to produce other NFS. Among all NFSs, the steamed soybean showed the highest H-ORAC value $(2.35 \pm 0.31 \mathrm{mmol} \mathrm{TE} / 100 \mathrm{~g})$. However, H-ORAC of the second low-processed product - edamame (young soybean seed), was much lower. It could be assumed that the difference was due to the content of the main antioxidative compounds, isoflavones, and soyasaponins. Indeed, it was reported that contents of daidzein and genistein in soybean were at 0.25 $-1.23 \mathrm{mg} / \mathrm{g}$ and $0.33-1.17 \mathrm{mg} / \mathrm{g}$, respectively [Wu et al., 2004]. On the other hand, edamame contained daidzein and genistein in the amounts of $0.11-0.55 \mathrm{mg} / \mathrm{g}$ and $0.16-0.62 \mathrm{mg} / \mathrm{g}$, respectively [Wu et al., 2004]. Moreover, as reported in the previous 

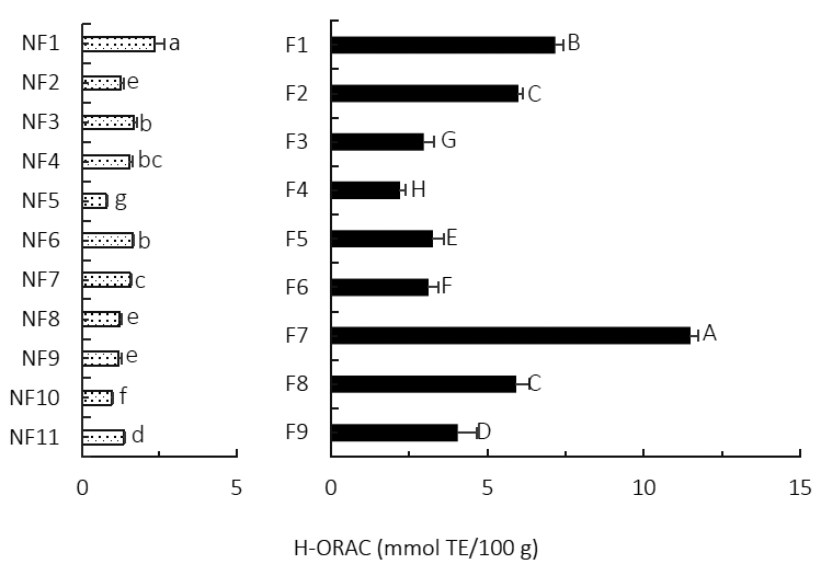

FIGURE 2. Hydrophilic-oxygen radical absorbance capacity (H-ORAC) of non-fermented (NF) and fermented (F) soyfoods. Details on the samples NF1-N11 and F1-F9 are provided in Table 1. Different letters present significant differences between samples $(p<0.05)$, lowercase letter for non-fermented soyfoods and uppercase letter for fermented soyfoods.

research, edamame contained much less soyasaponins than mature soybeans, and more active DDMP saponins were degraded to group B saponin during heating [Chitisankul et al., 2021]. Thus, the different antioxidant capacities of these low-processed samples might not be related to the food process but varied depending on the maturity of soybean.

For soymilk and soy beverages, four of five samples showed non-significant differences in H-ORAC values and only $\mathrm{H}$ -ORAC of modified soymilk (NF5) was significantly $(p<0.05)$ lower (Figure 2). Soy solid content of soymilk and soy beverages might play a significant role in their functional properties. On the report of product labeling, solid contents of regular (non-modified) soymilk (NF3), lipoxygenase-deficient soymilk (prepared from a soybean variety 'Kinusayaka' deficient in all of 3 lipoxygenase isozymes) (NF 4) and soy beverage (NF6) were 9 to $14 \mathrm{~g} / 100 \mathrm{~g}$ or more, whereas the modified soymilk (NF5) contained about $7 \mathrm{~g} / 100 \mathrm{~g}$. Therefore, it could be considered that soy solid content might play an important role in antioxidative capacity in soymilk and soy beverages. In turn, black soybean beverage (NF7) was expected to be a rich antioxidative compound source. The previous study revealed that black soybean, in addition to isoflavones and saponins, contained significant amounts of anthocyanins and proanthocyanidins [Xu et al., 2017; Zilic et al., 2019] and showed significantly higher antioxidative capacity than regular soybean [Chitisankul et al., 2019]. However, anthocyanins are unstable water-soluble components that could be degraded by enzymatic reaction and thermal treatment [Slavu(Ursu) et al., 2020]. The thermal treatment is generally required to preserve and process food for safety purposes. The traditional soymilk process might require thermal treatment at $99^{\circ} \mathrm{C}$ while steam-infusion treatment might facilitate a higher temperature of about $99-154^{\circ} \mathrm{C}$ [Johnson et al., 1981]. In the last group of NFS samples, tofu products showed the lowest peroxyl radical scavenging capacity, especially abura-age, twice-fried tofu (NF10) (Figure 2). There was no significant difference in $\mathrm{H}$ -ORAC between momen tofu (NF8) and silken tofu (NF9), with their $\mathrm{H}-\mathrm{ORAC}$ values being lower than that of nama-age, fried tofu (NF11).
In summary, there was a significant difference in H-ORAC values among each group of NFS soyfoods. It was considered that the slight difference found was mainly due to the difference in the isoflavone and saponin contents and the respective compositions caused by the difference in the food processing treatment such as thermal process, extraction, and coagulation. This indicates that the bioactive compounds may change upon food processing and that each food product requires a different treatment process. The chemical structures of active compounds such as isoflavones and soyasaponins have an important role in nutraceutical property in soyfood as mentioned above. Malonyl glycoside, which is the main component of soybean isoflavone, is unstable to heat and derived from malonyl glycoside to glycoside and further to aglycone upon thermal treatment [Kasuga et al., 2006; Toda et al., 2000]. DDMP saponin can be degraded to group B saponin by thermal treatment and group E saponins by lipoxygenase-induced radical reaction during grinding [Chitisankul et al., 2015]. It was considered that the non-fermented soybean foods had different antioxidative capacities due to their isoflavone and DDMP saponin compositions, depending on the processing treatment during the manufacturing process.

\section{Peroxyl radical scavenging capacity of fermented soybean food}

Fermented soyfoods (FS) could be categorized into four groups; natto (F1 and F2), tempeh (F3), miso (F4-F7), and soy sauce (F8 and F9). Among all FS samples, soy miso showed the highest H-ORAC value followed by natto, soy sauce, other types of miso (F5 and F6), and tempeh, respectively (Figure 2). In the comparison of NF and FS samples, all FS products had higher H-ORAC values than NFS samples. The H-ORAC value of FS samples ranged from 2.21 \pm 0.19 to $11.53 \pm 0.41 \mathrm{mmol} \mathrm{TE} / 100 \mathrm{~g}$ while whole soybean seed (NF1) had $2.35 \mathrm{mmol} \mathrm{TE} / 100 \mathrm{~g}$.

High antioxidative capacity of black soybean natto was expected because, as mentioned above, the seed coat of black soybean is rich in anthocyanins. Moreover, the previous research revealed that the H-ORAC value of black soybean was $5,870 \pm 115 \mu \mathrm{mol} \mathrm{TE} / 100 \mathrm{~g}$ while that of soybean was 4,369 $\pm 418 \mu \mathrm{mol}$ TE/100 g [Chitisankul et al., 2019]. However, the previous research also reported that isoflavone content of soybean was higher than that of black soybean [Chitisankul et al., 2019]. Thus, it could be considered that isoflavones make a significant contribution to the peroxyl radical scavenging capacity instead of anthocyanins in natto. In addition, the H-ORAC value of natto was 3-fold higher compared to steamed soybean. Therefore, it clearly showed that the fermented process of natto could induce nutraceutical properties as enhancing antioxidant capacity. The biotransformation of isoflavones plays an important role to enhance those functionalities. The consistent reports explained the glycoside conjugates of isoflavones could be converted to isoflavone aglycones by $\beta$-glucosidase of Bacillus subtilis during fermentation [Dajanta et al., 2009; Khosravi \& Razavi, 2021; Ping et al., 2012]. Hence, the enhanced antioxidative capacity of regular soybean natto compared to black soybean natto might be due to three reasons: regular soybean had a higher isoflavone content, aglycone isoflavones presented 
stronger antioxidative capacity than anthocyanins, and degradation of water-soluble anthocyanins during natto production resulted in functionality losses. Moreover, it was found that natto had a higher antioxidant capacity than steamed soybeans (Figure 2). The browning substances derived from the amino-carbonyl reaction [Ando et al., 2003] and antioxidant peptides released from proteins during fermentation [Sanjukta \& Rai, 2016; Tonolo et al., 2020] could additionally contribute to the antioxidant potential of natto.

Although soy-miso (F7) showed the highest antioxidant activity, other kinds of miso (F4-F6) presented much lower peroxyl radical scavenging capacity especially the sweet-white type of rice miso (F4) with $\mathrm{H}-\mathrm{ORAC}$ value of $2.21 \pm 0.19 \mathrm{mmol}$ $\mathrm{TE} / 100 \mathrm{~g}$ (Figure 2). In the consent report, the antioxidant properties of different kinds of miso were evaluated by determining 2,2-diphenyl-1-picrylhydrazyl (DPPH) radical and superoxide scavenging activities [Matsuo \& Hitomi, 2007]. The soy-miso presented the highest antioxidative capacity following dark-yellow rice miso, light yellow rice miso, and sweet-white rice miso. And barley miso showed similar property with dark-yellow rice miso. Moreover, it was reported that the DPPH radical scavenging ability and the superoxide scavenging activity were strongly positively correlated with the total isoflavone content and aglycone content, respectively [Matsuo \& Hitomi, 2007]. During Aspergillus spp. fermentation in miso, the $\beta$-glucosidase was produced and activated to hydrolyze glycoside isoflavone to aglycone type isoflavones [Yamabe et al., 2007; Yan et al., 2016]. Furthermore, during the malting process in soy miso, aglycones (daidzein and genistein) could be converted to $o$-dihydroxy -isoflavones (ODI) while rice miso and barley miso do not contain ODI [Esaki et al., 2001b]. It was reported that ODI also contribute to the antioxidant properties of miso, since the higher the amount of ODI, the higher the antioxidant capacity [Esaki et al., 2001a]. Additionally, the lower lightness $\left(L^{*}\right)$ of the miso sample was revealed as related to higher antioxidative capacity. It was speculated that the browning substance derived from the amino-carbonyl reaction during fermentation and aging could contribute to the peroxyl radical scavenging activity of miso. For soy sauce, the H-ORAC value of dark-colored soy sauce (F8) was higher than that of light-yellow soy sauce (F9) (Figure 2). Similarly to miso, soy sauce undergoes fermentation and aging which result in aglycone isoflavone production; but there was a low total isoflavone content in soy sauce [Toda et al., 2000]. Although the aglycone isoflavones were formed during the soy sauce production, they were detected as remaining in soy sauce cake, a by-product [Esaki et al., 2004]. However, a positive correlation was found between the ODI content of soy sauce and its antioxidative capacity, and it was reported that ODI also contributed to the antioxidative capacity of soy sauce [Esaki et al., 2002]. According to this evaluation, it is considered that the peroxyl radical scavenging activity of soy sauce, which was recognized by the ORAC value, is related to the browning substance derived from the amino-carbonyl reaction and ODI. Tempeh (F3) had a similar H-ORAC value as rice miso and barley miso, but lower than that of natto (Figure 2). $\beta$-Glucosidase produced by Rhizopus filamentous fungi, which is used for tempeh fermentation, hydrolyzes
TABLE 2. Color parameters of miso and soy sauces.

\begin{tabular}{lcc|c}
\hline Soybean foods & $L^{*}$ & $a^{*}$ & $b^{*}$ \\
\hline Rice miso: sweet-white type & $65.33 \pm 0.03^{\mathrm{a}}$ & $4.35 \pm 0.05^{\mathrm{d}}$ & $35.37 \pm 0.03^{\mathrm{c}}$ \\
Rice miso: light yellow type & $48.82 \pm 0.04^{\mathrm{b}}$ & $9.33 \pm 0.08^{\mathrm{a}}$ & $41.96 \pm 0.02^{\mathrm{a}}$ \\
Barley miso & $44.86 \pm 0.08^{\mathrm{c}}$ & $6.86 \pm 0.01^{\mathrm{b}}$ & $35.68 \pm 0.08^{\mathrm{b}}$ \\
Soy miso & $10.90 \pm 0.09^{\mathrm{d}}$ & $5.73 \pm 0.26^{\mathrm{c}}$ & $8.24 \pm 0.26^{\mathrm{d}}$ \\
\hline Dark soy sauce & 14.16 & 29.92 & 23.38 \\
Light soy sauce & 43.64 & 36.73 & 72.23 \\
\hline
\end{tabular}

Values for miso are expressed as mean \pm standard deviation $(n=3)$; for soy sauce value $n=1$. Different letters in column presented significant differences $(p<0.05) ; L^{*}$, lightness; $a^{*}$, redness; $b^{*}$, yellowness.

isoflavone glycosides into aglycones [Kameda et al., 2018]. It was suggested that the isoflavone aglycone produced during fermentation might contribute to the antioxidant properties of tempeh [Murakami et al., 1984].

\section{Color of fermented soybean food vs. peroxyl radical scavenging capacity}

The color parameters of long-term fermented soyfood samples, miso, and soy sauce were measured and $L^{*}, a^{*}$, and $b^{*}$ values are shown in Table 2 . The relationship of color parameters with antioxidative capacity was also evaluated. The $L^{*}$ value of miso decreased in order of sweet-white rice miso, light yellow rice miso, barley miso, and soy miso, the darkest miso. The $a^{*}$ value of light yellow rice miso was slightly higher than that of the other four miso samples. There was no significant difference in the $b^{*}$ value among miso samples, except soymiso which showed the lowest yellowness. On the other hand, dark soy sauce showed lower $L^{*}, a^{*}$, and $b^{*}$ values than light soy sauce. After $L^{*}, a^{*}, b^{*}$ value measurement, the correlations between these values and peroxyl radical scavenging capacity were found for miso and soy sauce. The results revealed that there were no significant correlations of H-ORAC values with $a^{*}$ and $b^{*}$ values. On the other hand, a strong negative correlation $(\mathrm{r}=-0.9747, p=0.0048)$ was found between the $L^{*}$ and H-ORAC values of miso samples and soy sauce, excluding soy miso (Figure 3 ). Therefore, the low $L^{*}$ value, darkness



FIGURE 3 . Correlation between lightness $\left(L^{*}\right)$ and hydrophilic-oxygen radical absorbance capacity (H-ORAC) of long-term fermented soyfoods; miso (F4-F7) and soy sauces (F8-F9). For code of samples see Table 1. The correlation coefficient ( $r$ ) and significance level ( $\mathrm{p}$ ) were calculated excluding soy miso (F7). 
of soy sauce or miso, might be an index of browning reagent contents, the amino-carbonyl reaction product which induced a higher H-ORAC value. The result was supported by other consistent reports. The correlations of $L^{*}$ value with both DPPH radical and superoxide scavenging activities of various miso paste samples were negative [Matsuo \& Hitomi, 2007]. In our study, soybean miso presented a significantly higher $\mathrm{H}-\mathrm{ORAC}$ value than the expected H-ORAC value based on the $L^{*}$ value (Figure 3 ). It could be explained that soybean miso had a higher ratio of soybean content than other kinds of miso, and the soybean miso production might be different too. Therefore, the strong peroxyl radical scavenging capacity of soybean miso might be due to the high content of isoflavones, saponins, ODI [Esaki et al., 2001a,b], and antioxidative peptides released from proteins [Sanjukta \& Rai, 2016].

\section{CONCLUSIONS}

Since the H-ORAC method is an in vitro method for measuring antioxidant capacity, its results might not directly reflect the antioxidative capacity in vivo, but it can be a simple primary screening tool for antioxidant capacity measurments in many soyfoods. Fermented soybean could promote higher antioxidant properties compared to non-fermented soyfood due to peroxyl radical scavenging capacity. The chemical structure of bioactive compounds in soybean plays an important role in the antioxidant properties of soyfood products. The technological processes could cause transformations of those healthy functional compounds and thus enhance or diminish the antioxidative capacity. The experiment demonstrated that derived isoflavone as aglycone could promote higher antioxidative activity while derived soyasaponins, such as group A and group B saponins, showed lower activity than DDMP-saponin. According to the H-ORAC value of soyfood sample, fermented soyfood presented the highest antioxidant capacity which was due to a high aglycone isoflavone content. Furthermore, it was also considered that peptides and free amino acids produced by aging also contributed to the antioxidant capacity of fermented soybean foods. Among all processed soyfood samples, the soy-miso sample had the highest H-ORAC value followed by natto samples. Eventually, considering the serving size and antioxidant capacity, natto is the most effective product compared to miso or soy sauce having a high salt content. However, long-term fermented soyfood should still be in the focus of interest to researchers as health promoting food. There is a lack of data on their minor bioactive components such as antioxidant peptides and ODI. Thus, these minor components should be considered for further study.

\section{RESEARCH FUNDING}

This research was partly supported by "JSPS RONPAKU (Dissertation Ph.D.) Program 2012 (Grant ID No. THA - 11206)" and "Kasetsart University Research and Development Institute".

\section{CONFLICT OF INTERESTS}

Authors declare no conflict of interests.

\section{ORCID IDs}

W.T. Chitisankul https://orcid.org/0000-0003-2556-7698

Ch. Tsukamoto https://orcid.org/0000-0002-6437-2140

\section{REFERENCES}

1. Ando, M., Harada, K., Kitao, S., Kobayashi, M., Tamura, Y. (2003). Relationship between peroxyl radical scavenging capability measured by the chemiluminescence method and an aminocarbonyl reaction product in soy sauce. International Journal of Molecular Medicine, 12(6), 923-928.

https://doi.org/10.3892/ijmm.12.6.923

2. Arora, A., Nair, M.G., Strasburg, G.M. (1998). Antioxidant activities of isoflavones and their biological metabolites in a liposomal system. Archives of Biochemistry and Biophysics, 356(2), 133-141.

https://doi.org/10.1006/abbi.1998.0783

3. Bultosa, G. (2016). Functional foods: Overview. In C. Wrigley, H. Corke, K. Seetharaman, J. Faubion (Eds.), Encyclopedia of Food Grains (Second Edition), Academic Press, Oxford, pp. $1-10$.

https://doi.org/10.1016/B978-0-12-394437-5.00071-1

4. Carrera, C.S., Seguin, P. (2016). Factors affecting tocopherol concentrations in soybean seeds. Journal of Agricultural and Food Chemistry, 64(50), 9465-9474.

https://doi.org/10.1021/acs.jafc.6b03902

5. Chang, C.-T., Hsu, C.-K., Chou, S.-T., Chen, Y.-C., Huang, F.-S., Chung, Y.-C. (2009). Effect of fermentation time on the antioxidant activities of tempeh prepared from fermented soybean using Rhizopus oligosporus. International Journal of Food Science \& Technology, 44(4), 799-806.

https://doi.org/10.1111/j.1365-2621.2009.01907.x

6. Chitisankul, W.T., Itabashi, M., Son, H., Takahashi, Y., Ito, A., Varanyanond, W., Tsukamoto, C. (2021). Soyasaponin composition complexities in soyfoods relating nutraceutical properties and undesirable taste characteristics. LWT - Food Science and Technology, 146, art. no. 111337.

https://doi.org/10.1016/j.Iwt.2021.111337

7. Chitisankul, W.T., Murakami, M., Tsukamoto, C., Shimada, K. (2019). Effects of long-term soaking on nutraceutical and taste characteristic components in Thai soybeans. LWT-Food Science and Technology, 115, art no. 108432.

https://doi.org/10.1016/j.lwt.2019.108432

8. Chitisankul, W.T., Shimada, K., Omizu, Y., Uemoto, Y., Varanyanond, W., Tsukamoto, C. (2015). Mechanism of DDMP-saponin degradation and maltol production in soymilk preparation. LWT - Food Science and Technology, 64(1), 197-204. https://doi.org/10.1016/j.Iwt.2015.05.023

9. Dajanta, K., Chukeatirote, E., Apichartsrangkoon, A., Frazier, R. (2009). Enhanced aglycone production of fermented soybean products by Bacillus species. Acta Biologica Szegediensis, 53, 93-98.

10. Esaki, H., Kawakishi, S., Inoue, T., Osawa, T. (2001a). Potent antioxidative $o$-dihydroxyisoflavones in soybean pastes and their antioxidative activities. Nippon Shokuhin Kagaku Kogaku Kaishi, 48(1), 51-57.

https://doi.org/10.3136/nskkk.48.51 
11. Esaki, H., Osawa, T., Kawakishi, S. (2002). Potent antioxidative $o$-dihydroxyisoflavones in soy sauces and their antioxidative activities. Nippon Shokuhin Kagaku Kogaku Kaishi, 49(7), 476-483. https://doi.org/10.3136/nskkk.49.476

12. Esaki, H., Watanabe, R., Hishikawa, N., Osawa, T., Kawakishi, S. (2004). Utility of isoflavone preparations from soy sauce cake as antioxidant materials. Nippon Shokuhin Kagaku Kogaku Kaishi, 51(1), 47-53.

https://doi.org/10.3136/nskkk.51.47

13. Esaki, H., Watanabe, R., Masuda, H., Osawa, T., Kawakishi, S. (2001b). Formations and changes of $o$-dihydroxyisoflavones during production of soybean pastes. Nippon Shokuhin Kagaku Kogaku Kaishi, 48(3), 189-195.

https://doi.org/10.3136/nskkk.48.189

14. Han, R.-M., Tian, Y.-X., Liu, Y., Chen, C.-H., Ai, X.-C., Zhang, J.-P., Skibsted, L.H. (2009). Comparison of flavonoids and isoflavonoids as antioxidants. Journal of Agricultural and Food Chemistry, 57(9), 3780-3785.

https://doi.org/10.1021/jf803850p

15. Johnson, L.A., Deyoe, C.W., Hoover, W.J. (1981). Yield and quality of soymilk processed by steam-infusion cooking. Journal of Food Science, 46(1), 239-248.

https://doi.org/10.1111/j.1365-2621.1981.tb14572.x

16. Kähkönen, M.P., Heinonen, M. (2003). Antioxidant activity of anthocyanins and their aglycons. Journal of Agricultural and Food Chemistry, 51 (3), 628-633. https://doi.org/10.1021/jf025551i

17. Kameda, T., Aoki, H., Yanaka, N., Kumrungsee, T., Kato, N. (2018). Production of isoflavone aglycone-enriched tempeh with Rhizopus stolonnifer. Food Science and Technology Research, 24(3), 493-499.

https://doi.org/10.3136/fstr.24.493

18. Kasuga, A., Ogiwara, E., Aoyagi, Y., Kimura, H. (2006). Changes in isoflavone content of soybeans during heating process. Nippon Shokuhin Kagaku Kogaku Kaishi, 53(7), 365-372.

https://doi.org/10.3136/nskkk.53.365

19. Khosravi, A., Razavi, S.H. (2021). Therapeutic effects of polyphenols in fermented soybean and black soybean products. Journal of Functional Foods, 81, art. no. 104467.

https://doi.org/10.1016/j.jff.2021.104467

20. Kim, M.S., Jung, Y.S., Jang, D., Cho, C.H., Lee, S.-H., Han, N.S., Kim, D-O. (2022). Antioxidant capacity of 12 major soybean isoflavones and their bioavailability under simulated digestion and in human intestinal Caco-2 cells. Food Chemistry, 374, art. no. 131493.

https://doi.org/10.1016/j.foodchem.2021.131493

21. Lee, C.H., Yang, L., Xu, J.Z., Yeung, S.Y.V., Huang, Y., Chen, Z.-Y. (2005). Relative antioxidant activity of soybean isoflavones and their glycosides. Food Chemistry, 90(4), 735-741. https://doi.org/10.1016/j.foodchem.2004.04.034

22. Long, L.H., Kwee, D.C.T., Halliwell, B. (2000). The antioxidant activities of seasonings used in Asian cooking. Powerful antioxidant activity of dark soy sauce revealed using the ABTS assay. Free Radical Research, 32(2), 181-186. https://doi.org/10.1080/10715760000300181

23. Matsuo, M., Hitomi, E. (2007). Changes in antioxidant activity of miso by variety, cooking and added spices. Nippon Shokuhin Kagaku Kogaku Kaishi, 54(11), 503-508.

https://doi.org/10.3136/nskkk.54.503
24. Murakami, H., Asakawa, T., Terao, J., Matsushita, S. (1984). Antioxidative stability of tempeh and liberation of isoflavones by fermentation. Agricultural and Biological Chemistry, 48(12), 2971-2975.

https://doi.org/10.1080/00021369.1984.10866635

25. Omizu, Y., Tsukamoto, C., Chettri, R., Tamang, J.P. (2011). Determination of saponin contents in raw soybean and fermented soybean foods of India. Journal of Scientific \& Industrial Research, 70, 533-538.

26. Ou, B., Hampsch-Woodill, M., Prior, R. (2001). Development and validation of an improved oxygen radical absorbance capacity assay using fluorescein as the fluorescent probe. Journal of $A g$ ricultural and Food Chemistry, 49(10), 4619-4626. https://doi.org/10.1021/jf0105860

27. Ping, S.P., Shih, S.C., Rong, C.T., King, W.Q. (2012). Effect of isoflavone aglycone content and antioxidation activity in natto by various cultures of Bacillus Subtilis during the fermentation period. Journal of Nutrition \& Food Sciences, 2(7), art. no. 1000153. https://doi.org/10.4172/2155-9600.1000153

28. Pizzino, G., Irrera, N., Cucinotta, M., Pallio, G., Mannino, F., Arcoraci, V., Squadrito, F., Altavilla, D., Bitto, A. (2017). Oxidative stress: Harms and benefits for human health. Oxidative Medicine and Cellular Longevity, 2017, art. no. 8416763.

https://doi.org/10.1155/2017/8416763

29. Ruiz-Larrea, M.B., Mohan, A.R., Paganga, G., Miller, N.J., Bolwell, G.P., Rice-Evans, C.A. (1997). Antioxidant activity of phytoestrogenic isoflavones. Free Radical Research, 26(1), 63-70. https://doi.org/10.3109/10715769709097785

30. Sanjukta, S., Rai, A.K. (2016). Production of bioactive peptides during soybean fermentation and their potential health benefits. Trends in Food Science \& Technology, 50, 1-10. https://doi.org/10.1016/j.tifs.2016.01.010

31. Setchell, K., Brown, N., Zimmer-Nechemias, L., Brashear, W., Wolfe, B., Kirschner, A., Heubi, J. (2002). Evidence for lack of absorption of soy isoflavone glycosides in humans, supporting the crucial role of intestinal metabolism for bioavailability. The American Journal of Clinical Nutrition, 76(2), 447-453. https://doi.org/10.1093/ajcn/76.2.447

32. Slavu(Ursu), M., Aprodu, I., Milea, S.A., Enachi, E., Râpeanu, G., Bahrim, G.E., Stanciuc, N. (2020). Thermal degradation kinetics of anthocyanins extracted from purple maize flour extract and the effect of heating on selected biological functionality. Foods, 9(11), art. no. 1593. https://doi.org/10.3390/foods9111593

33. Toda, T., Sakamoto, A., Takayanagi, T., Yokotsuka, K. (2000). Changes in isoflavone compositions of soybean foods during cooking process. Food Science and Technology Research, 6(4), 314-319. https://doi.org/10.3136/fstr.6.314

34. Tonolo, F., Moretto, L., Grinzato, A., Fiorese, F., Folda, A., Scalcon, V., Ferro, S., Arrigoni, G., Bellamio, M., Feller, E., Bindoli, A., Marin, O., Rigobello, M.P. (2020). Fermented soy-derived bioactive peptides selected by a molecular docking approach show antioxidant properties involving the Keap1/Nrf2 pathway. Antioxidants, 9(12), art. no. 1306. https://doi.org/10.3390/antiox9121306

35. Wu, Q., Wang, M., Sciarappa, W.J., Simon, J.E. (2004). LC/UV/ ESI-MS analysis of isoflavones in edamame and tofu soybeans. Journal of Agricultural Food Chemistry, 52(10), 2763-2769. https://doi.org/10.1021/jf035053p 
36. Xu, J.L., Shin, J.-S., Park, S.K., Kang, S., Jeong, S.-C., Moon, J.-K., Choi, Y. (2017). Differences in the metabolic profiles and antioxidant activities of wild and cultivated black soybeans evaluated by correlation analysis. Food Research International, 100(Part 2), 166-174.

https://doi.org/10.1016/j.foodres.2017.08.026

37. Yamabe, S., Kobayashi-Hattori, K., Kaneko, K., Endo, H., Takita, T. (2007). Effect of soybean varieties on the content and composition of isoflavone in rice-koji miso. Food Chemistry, 100(1), 369-374.

https://doi.org/10.1016/j.foodchem.2005.09.061

38. Yan, F.-Y., Xia, W., Zhang, X.-X., Chen, S., Nie, X.-Z., Qian, L.-C. (2016). Characterization of $\beta$-glucosidase from Aspergillus terreus and its application in the hydrolysis of soybean isoflavones. Journal of Zhejiang University Science B, 17(6), 455-464. https://doi.org/10.1631/jzus.B1500317

39. Yoshiki, Y., Okubo, K. (1995). Active oxygen scavenging activity of DDMP (2,3-Dihydro-2,5-dihydroxy-6-methyl-4H-pyran- -4-one) saponin in soybean seed. Bioscience, Biotechnology, and Biochemistry, 59(8), 1556-1557.

https://doi.org/10.1271/bbb.59.1556

40. Yoshiki, Y., Kahara, T., Okubo, K., Sakabe, T., Yamasaki, T. (2001). Superoxide- and 1,1-diphenyl-2-picrylhydrazyl radical-scavenging activities of soyasaponin $\beta \mathrm{g}$ related to gallic acid. Bioscience, Biotechnology, and Biochemistry, 65(10), 2162-2165. https://doi.org/10.1271/bbb.65.2162

41. Zhong, Y., Shahidi, F. (2015). Methods for the assessment of antioxidant activity in foods. In F. Shahidi (Ed.), Handbook of Antioxidants for Food Preservation, Woodhead Publishing, Cambridge, pp. 287-333.

42. Zilic, S., Dodig, D., Vancetovic, J., Grcic, N., Peric, V., Titan, P., Maksimovic, V. (2019). Composition of anthocyanins in colored grains and the relationship of their non-acylated and acylated derivatives. Polish Journal of Food and Nutrition Sciences, 69(2), $137-146$.

https://doi.org/10.31883/pjfns-2019-105100 